\title{
Perianchor Cyst Formation Is Similar Between All-Suture and Conventional Suture Anchors Used for Arthroscopic Rotator Cuff Repair in the Same Shoulder
}

\author{
Elliott W. Cole, M.D., M.P.H., Brian C. Werner, M.D., and Patrick J. Denard, M.D.
}

Purpose: The purpose of this study was to compare perianchor cyst formation between soft and hard suture anchors placed in the same patient 1 year after arthroscopic rotator cuff repair (ARCR). Methods: This study reviewed patients who underwent primary ARCR using a "hybrid" technique using at least one soft anchor (FiberTak, Arthrex, Naples, FL) and one hard anchor (SwiveLock) placed in the same shoulder between January 1, 2018 and December $31,2018$. Magnetic resonance imaging was obtained at minimum 1-year postoperative to assess cyst formation (perianchor fluid signal) and rotator cuff healing. Range of motion (ROM) and patient-reported outcome measures (PROMs) were evaluated at baseline and 1-year follow up. PROMs included visual analog scale pain score, Simple Shoulder Test score, American Shoulder and Elbow Surgeon (ASES) score. Results: Nineteen patients with a combined 45 hard and 26 soft suture anchors were available for follow-up at a mean of 20 months postoperatively. There was a higher proportion of grade 1 fluid signal changes in the hard anchor group compared to the soft group $(62.2 \%$ to $7.7 ; P<.001)$; however, there was no difference in the incidence of cyst formation (grade 2 or 3 changes) between groups $(13.3 \%$ vs $3.8 \% ; P=.251)$. There was also no difference in the rate of cyst formation between biocomposite and polyether-ether-ketone-type hard anchors $(18.2 \%$ vs $0 \% ; P=.113)$ or between anchors placed at the greater and lesser tuberosities (10.2\% vs $5.3 \%$, $P=.519)$. Conclusion: Hard suture anchors showed increased fluid signal compared to soft suture anchors at short-term follow-up after ARCR, but there was no difference in cyst formation between anchor types.

\section{Introduction}

$\mathbf{T}$ he surgical management of rotator cuff tears has evolved with improved understanding of shoulder biology and biomechanics. ${ }^{1}$ Transosseous bone tunnels were historically used to repair the rotator cuff footprint but have largely been replaced by suture anchors with

Department of Orthopaedic $\theta$ Rehabilitation, Oregon Health $\theta$ Science University, Portland, Oregon, U.S.A. (E.W.C., P.J.D.); Department of Orthopaedic Surgery, University of Virginia, Charlottesville, Virginia, U.S.A. (B.C.W.); and Oregon Shoulder Institute, Medford, Oregon, U.S.A. (P.J.D.).

The authors report the following potential conflicts of interest or sources of funding: B.W. reports consulting fees and royalties from Arthrex. P.J.D. reports consulting fees and grants from Arthrex. Full ICMJE author disclosure forms are available for this article online, as supplementary material.

Received August 17, 2021; accepted January 14, 2022.

Address correspondence to Patrick J. Denard, M.D., Southern Oregon Orthopedics, 2780 E. Barnett Rd., Suite 200, Medford, OR, 97530, U.S.A. E-mail:pjdenard@gmail.com

(C) 2022 THE AUTHORS. Published by Elsevier Inc. on behalf of the Arthroscopy Association of North America. This is an open access article under the CC BY-NC-ND license (http://creativecommons.org/licenses/by-nc-nd/4.0/). 2666-061X/211155

https://doi.org/10.1016/j.asmr.2022.01.007 superior biomechanical properties. ${ }^{2}$ Conventional "hard" suture anchors have been made of metal, PEEK, or absorbable composite materials. The original metal anchors provided excellent fixation, but they were known to cause artifacts on postoperative magnetic resonance imaging (MRI) assessment, as well as possible tissue damage in the event of pullout. Biocomposite and PEEK anchors were developed to overcome these disadvantages, but these anchors have been associated with increased perianchor fluid signal and osteolysis. ${ }^{3,4}$ All-suture "soft" suture anchors are a more recent development that theoretically offer the same biomechanical advantages of other hard anchors with the added benefit of smaller size, thereby preserving bone stock and increasing the rotator cuff footprint area available for tendon healing.

All-suture anchors are commonly used in the glenoid for labral repair. Although there were concerns that earlier generations of soft anchors may be susceptible to cyst formation and early failure, ${ }^{5,6}$ recent studies have shown all-suture and conventional anchors to be comparable fixation methods in the glenoid, with 
similar biomechanical properties and clinical outcomes. ${ }^{7-11}$

Soft suture anchors are increasingly being used in rotator cuff repair, but few studies have evaluated their performance. A recent systematic review of all-suture anchors included only one study examining their outcomes in rotator cuff repair, and although this study showed good results at 1 year postoperatively, it highlights the fact that there are limited data about soft anchor performance in rotator cuff surgery. ${ }^{8,12}$ A few studies have directly compared soft anchor and hard anchor performance in rotator cuff repair, but little is known about how the two anchor types perform within the same patient. The senior author (P.D.) often uses a hybrid technique in which both soft and hard anchors are used to repair an individual rotator cuff, providing a unique opportunity to compare the MRI appearance of these two anchor types.

The purpose of this study was to compare perianchor cyst formation between soft and hard suture anchors placed in the same patient 1 year after arthroscopic rotator cuff repair (ARCR). The hypothesis was that there would be no difference in MRI appearance based on anchor type.

\section{Materials and Methods}

\section{Patient Selection}

After Institutional Review Board (IRB) approval was obtained, a retrospective review was performed of data collected in a prospective registry (Surgical Outcomes System (SOS), Arthrex, Inc, Naples, FL) at a single institution. This registry was reviewed to identify patients who underwent ARCR by a single surgeon (P.J.D.) between January 1, 2018 and Dec 31, 2018. Inclusion criteria included full-thickness rotator cuff tears, primary surgery, minimum follow-up of 1 year, and a hybrid repair consisting of at least 1 all-suture anchor and 1 conventional suture anchor placed in the same shoulder. Exclusion criteria included partial tears and revision repairs.

An analysis was performed prior to recruitment to determine the number of hard and soft anchors that would need to be analyzed to detect a difference in the perianchor fluid signal between the two anchor types. In order to detect a mean fluid grade difference between the hard and soft anchor groups of .5 using the group standard deviation of .6 with a power of $80 \%$ and $\alpha=.05$, it was determined that a minimum of 23 anchors were needed in each group (23 all-suture anchors, 23 conventional anchors). Patients who met study criteria were then recruited consecutively to undergo a postoperative MRI until the minimum number of hard and soft anchors required for analysis had been imaged.
Table 1A. Demographics

\begin{tabular}{lc}
\hline Demographics & Mean $(\mathrm{SD})$ or $n(\%)$ \\
\hline Age & $67.6(8.3)$ \\
Sex & \\
F & $10(52.6)$ \\
M & $9(47.4)$ \\
Dominant Arm & \\
$\quad$ Yes & $13(68.4)$ \\
No & $6(31.6)$ \\
Tobacco Use & \\
Yes & $9(47.4)$ \\
No & $10(52.6)$ \\
\hline
\end{tabular}

\section{Surgical Procedure}

All ARCRs were performed by a single surgeon (P.J.D.) using a hybrid technique, in which at least 1 allsuture anchor (FiberTak, Arthrex, Inc.) and 1 conventional suture anchor (SwiveLock, Arthrex, Inc.) were used. Both polyether ether ketone (PEEK) and biocomposite ( $85 \%$ poly-L-lactic acid (PLLA), 15\% betatricalcium phosphate) conventional suture anchor types were used. The choice of which conventional suture anchor to use was based on surgeon preference. Acromioplasty was performed in $100 \%(19 / 19)$ of patients. Biceps tenodesis was performed in $79 \%(15 / 19)$ of patients with a SwiveLock anchor using an onlay technique, in which the anchor can be used for biceps tenodesis alone or for simultaneous biceps tenodesis and supraspinatus or subscapularis repair. Postoperatively, patients were immobilized in a sling for 4 to 6 weeks on the basis of tear size. Passive range of motion (ROM) exercises were initiated after sling removal, followed by strengthening at 8 to 12 weeks postoperatively.

\section{Clinical Evaluation}

ROM and patient-reported outcome measures (PROMS) were recorded preoperatively, and at a minimum one-year postoperation, ROM measures were evaluated by the treating surgeon (P.J.D.). These measures included forward flexion (FF), external rotation (ER), and internal rotation to the nearest spinal level (IR). PROMs included the American Shoulder and Elbow Surgeons Score (ASES), Subjective shoulder value (SSV), and visual analog scale (VAS) for pain.

\section{Imaging Analysis}

An MRI of the operative shoulder was obtained at a minimum of 1 year postoperatively to evaluate rotator cuff healing and anchor appearance. The MRI protocol was as follows:

1. Tl turbo spin echo (TSE) coronal: 20 slices; repetition time (TR), $550 \mathrm{~ms}$; echo time (TE), $11 \mathrm{~ms}$; voxel size, $.6 \times .6 \times 2.5 \mathrm{~mm}$; acquisition time $(\mathrm{TA}), \mathrm{l}: 46$;

2. T2 BLADE TSE coronal: 20 slices; TR, 2,450 ms; TE, $52 \mathrm{~ms}$; fat saturation (FATSAT), voxel size: $.5 \times .5 \times$ $2.5 \mathrm{~mm}$; TA, 4:11; 
Table 1B. Clinical Data: PROMs

\begin{tabular}{lccc}
\hline & Preoperative & Postoperative & $P$ Value \\
\hline VAS pain & $5.7(2.4)$ & $0.7(1.2)$ & $P<.001$ \\
ASES & $42.4(19.6)$ & $92.6(9.6)$ & $P<.001$ \\
SSV & $31.3(25.7)$ & $94.1(6.0)$ & $P<.001$ \\
FF (active) & $149(20)$ & $153(17)$ & $P=.511$ \\
ER0 (active) & $57(15)$ & $61(9)$ & $P=.327$ \\
IR Spinal level (active) & L4 (3) & L2 $(3)$ & $P=.047$ \\
\hline
\end{tabular}

Continuous variables listed as mean (SD), categorical variables listed as number $(\%)$.

ASES, American Shoulder and Elbow Surgeons Shoulder score; ER0, (active)-active external rotation with arm at side; FF (active), active forward flexion; IR, spinal level (active)-active internal rotation to average level obtained; PROM, patient-reported outcome measures; ROM, range of motion; SD, standard deviation; SSV, Subjective Shoulder Value; VAS pain, visual analog pain score.

3. T2 BLADE TSE sagittal: 30 slices; TR, 6,620 ms; TE, $103 \mathrm{~ms}$; FATSAT, voxel size: $.5 \times .5 \times 2.5 \mathrm{~mm}$; TA, 3:53; and

4. T2 BLADE TSE axial: 24 slices; TR, 5,300 ms; TE, 103 ms; FATSAT, voxel size: $.5 \times .5 \times 2.5 \mathrm{~mm}$; TA, $4: 48$

All images were reviewed by 2 independent examiners (P.J.D., E.W.C.) who rectified any discrepancies by mutual agreement. First, the integrity of the cuff repair was evaluated. An intact cuff was defined as maintenance of the insertion into the footprint, and an unhealed cuff was defined as a discontinuity at the footprint.

Second, the fluid signal around each anchor was evaluated on multiple T2-weighted images (coronal and sagittal) and graded 0-3 using the method previously described by Kim et al. ${ }^{13}$ : grade 0 , no fluid signal around the anchor; grade 1, minimal fluid collections; grade 2, local collection of fluid; grade 3, fluid collection around the entire length of the anchor, with diameter less than twice the anchor diameter. If no fluid signal around the anchor was observed, an increased signal on intermediary T2 images was scored similarly as the fluid signal. Similar to prior studies, we considered grade 2 and 3 changes to represent the presence of a perianchor bone cyst. $^{14}$

Third, the integrity of the cuff repair was graded 1-5 using the method developed by Sugaya et al. ${ }^{15}$ : grade 1 , normal thickness and intact; grade 2, increasing signal on T2 and intact; grade 3, loss of thickness without tear; grade 4 , presence of minor discontinuity (or high signal intensity area) in more than one slice of each of the standard T2-weighted images, suggesting a small retear; or grade 5, presence of a major discontinuity (or high signal intensity area) in more than one slice of each of the standard T2-weighted images, suggesting a medium or large retear. Grades 1-3 were considered healed, and grades 4 and 5 were considered a retear.

\section{Statistical Analysis}

Statistical analysis was performed using SPSS version 27 (IBM, Armonk, NY). Continuous data were described by mean and standard deviation and categorical data by count and percentage. Differences in preoperative and 1-year postoperative ROM and functional outcome scores were evaluated with a paired $t$ test. An unpaired $t$-test was used to compare the mean perianchor fluid signal between soft and hard (PEEK and biocomposite) anchors, between PEEK and biocomposite hard anchors, and between anchors placed at the greater tuberosity and lesser tuberosity. For all comparisons, $P<.05$ was considered statistically significant.

\section{Results}

Our review yielded 112 patients who met study criteria. A total of 19 consecutive patients with a combined 45 hard anchors (PEEK and biocomposite) and 26 soft anchors were enrolled and underwent MRI at a mean follow up of 20.4 months (range: 13-24). There were 33 biocomposite anchors and 12 PEEK anchors in the hard anchor group, while 49 anchors were placed in the greater tuberosity, and 19 anchors were placed in the lesser tuberosity. Of the 15 anchors used for biceps tenodesis, 3 anchors were used for biceps tenodesis alone, while 12 were used for simultaneous biceps tenodesis and cuff repair (either subscapularis or anterior supraspinatus tendon). The supraspinatus was repaired in all 19 patients, and the subscapularis was repaired in 13 patients. The mean age was 67.6 years (range: 39-78). Baseline characteristics and clinical outcomes are summarized in Table 1, A and B.

Table 2. Postoperative MRI Anchor Fluid Signal Comparison: Hard Versus Soft Anchors

\begin{tabular}{|c|c|c|c|c|c|}
\hline & \multicolumn{2}{|c|}{ Hard Anchors $(n=45)$} & \multicolumn{2}{|c|}{ Soft Anchors $(n=26)$} & \multirow[b]{2}{*}{$P$} \\
\hline & $n$ & $\%$ & $n$ & $\%$ & \\
\hline Grade 0 & 11 & $24.4 \%$ & 23 & $88.5 \%$ & $<.001$ \\
\hline Grade 1 & 28 & $62.2 \%$ & 2 & $7.7 \%$ & $<.001$ \\
\hline Grade 2 & 6 & $13.3 \%$ & 1 & $3.8 \%$ & .251 \\
\hline \multirow[t]{2}{*}{ Grade 3} & 0 & $0.0 \%$ & 0 & $0.0 \%$ & $\mathrm{n} / \mathrm{a}$ \\
\hline & Mean & S.D. & Mean & S.D. & \\
\hline Mean Grade & .89 & .60 & .15 & .46 & $<.001$ \\
\hline
\end{tabular}




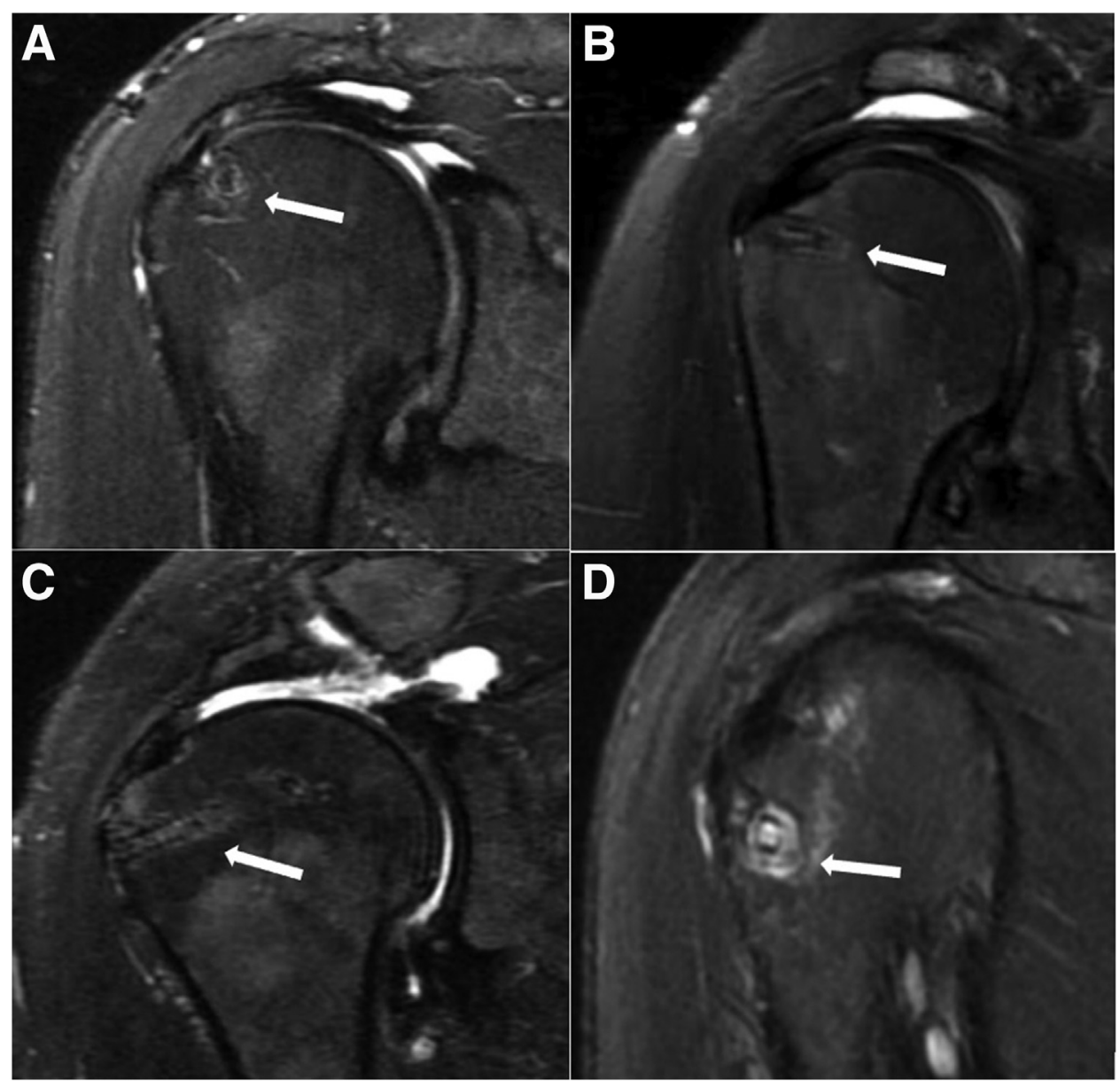

Fig 1. T2-weighted fat-saturated magnetic resonance imaging (MRI) images demonstrating grades 0-2 fluid signal changes. All images are right shoulders in the coronal plane with white arrows identifying suture anchor position. (A) Grade 0 (soft anchor): no fluid signal around the anchor. (B) Grade 0 (hard anchor): no fluid signal around the anchor. (C) Grade 1 (hard anchor): minimal fluid collection. (D) Grade 2 (hard anchor): local collection of fluid (cyst).

\section{Imaging Outcomes}

MRI appearance, according to hard and soft anchor types, are summarized in Table 2. A higher proportion of hard anchors demonstrated grade 1 fluid signal compared to the soft anchor group $(62.2 \%$ to $7.7 \%, P<$ $.001)$. There was no difference in the rate of cyst formation (grade 2 fluid signal) between hard and soft anchor groups ( $13.3 \%$ vs $3.8 \% ; P=.251$ ) (Fig 1 ). No anchors in either group had grade 3 fluid signal.

Subgroup analysis of the hard anchor group by anchor composition are summarized in Table 3. There was no difference in the proportion of anchors with grade 1 fluid signal between the biocomposite and PEEK anchor groups $(57.6 \%$ vs $75.0 \% ; P=.286)$. There was also no difference in the rate of cyst formation (grade 2 fluid signal) between biocomposite and PEEK anchor groups (18.2\% vs $0 \% ; P=.113$ ) No anchors in either group exhibited grade 3 changes.

MRI appearance of anchors based on tuberosity location is summarized in Table 4. There was no difference in the proportion of anchors with grade 1 fluid signal between anchors placed in the greater and lesser tuberosities $(38.8 \%$ vs $47.4 \% ; P=.518)$ There was also no difference in the rate of cyst formation (grade 2 fluid signal) between anchors placed in the greater and lesser tuberosities ( $10.2 \%$ vs $5.3 \% ; P=.519)$. Again, no anchors in either group demonstrated grade 3 changes.

The supraspinatus was considered healed (Sugaya $0-3)$ in $84.2 \%(16 / 19)$ of patients and to have a retear (Sugaya $4-5$ ) in $15.8 \%$ of patients (3/19). The subscapularis was considered healed (Sugaya 0-3) in 100\% (13/13) of patients, with no retears (Sugaya 4-5).

\section{Discussion}

The primary finding of our study is that 1 year after ARCR, there was no difference in the rate of cyst formation between hard and soft anchors in the same shoulder. Most of the soft suture anchors showed no perianchor fluid signal $(88.5 \%)$, while most of the hard anchors showed some degree of perianchor fluid signal $(75.5 \%)$. However, cyst formation rates were similar between hard and soft anchor groups (13.3\% vs 3.8\%; $P=.251)$.

Our finding of similar rates of perianchor cyst formation between conventional and all-suture suture anchors in ARCR is in line with prior findings. 
Table 3. Postoperative MRI Anchor Fluid Signal Comparison: PEEK Versus Composite Hard Anchors

\begin{tabular}{|c|c|c|c|c|c|}
\hline & \multicolumn{2}{|c|}{ Biocomposite $(n=33)$} & \multicolumn{2}{|c|}{$\operatorname{PEEK}(n=12)$} & \multirow[b]{2}{*}{$P$} \\
\hline & $n$ & $\%$ & $n$ & $\%$ & \\
\hline$\overline{\text { Grade } 0}$ & 8 & $24.2 \%$ & 3 & $25.0 \%$ & .958 \\
\hline Grade 1 & 19 & $57.6 \%$ & 9 & $75.0 \%$ & .286 \\
\hline Grade 2 & 6 & $18.2 \%$ & 0 & $0.0 \%$ & .113 \\
\hline \multirow[t]{2}{*}{ Grade 3} & 0 & $0 \%$ & 0 & $0 \%$ & $\mathrm{n} / \mathrm{a}$ \\
\hline & Mean & S.D. & Mean & S.D. & \\
\hline Mean Grade & 0.94 & 0.65 & 0.75 & 0.43 & .354 \\
\hline
\end{tabular}

Dividing line between Grades 1 and 2 represents the transition from minimal perianchor fluid signal (Grade 1) to cyst formation (Grades 2 and 3). n, number, S.D. standard deviation.

Ro et al. ${ }^{14}$ compared MRI perianchor fluid signal following single-row repairs in patients with either 137 all-suture anchors (Y-Knot RC, ConMed, Utica, NY), 36 biocomposite anchors (Healix Advance BR, DePuy Mitek, Raynham, MA), or 40 PEEK anchors (Helicoil PK, Smith \& Nephew, Andover, MA) and found no significant difference in cyst formation (grade 2 or above) between the three suture types.

We also found no difference in postoperative MRI appearance between PEEK and biocomposite hard anchors. Haneveld et al. ${ }^{3}$ compared the MRI perianchor fluid appearance of 36 patients who underwent rotator cuff repair: 16 with 100\% PLLA (Bio-Corkscrew FT, Arthrex, Inc.) and 20 with PEEK anchors (Corkscrew FT, Arthrex, Inc.). At an average of 28 months postoperatively, they found a significantly greater amount of perianchor fluid signal in the PLLA anchor group. In the current study, biocomposite anchors were used that consisted of $85 \%$ PLLA and $15 \% \beta$-tricalcium phosphate. We observed no difference between the 33 biocomposite and 12 PEEK anchors. Although the numbers are small, the lack of differences could be a reflection of the biocomposite anchor type. ${ }^{16}$ Studies comparing PEEK and biocomposite anchors used in the glenoid for labral repair have previously shown similar rates of cyst formation, ${ }^{17}$ and our findings suggest that PLLA biocomposite and PEEK anchors may have similar rates of cyst formation in the humeral tuberosities for rotator cuff repair as well.

Additionally, there was no difference in perianchor fluid signal between anchors placed in the greater and lesser tuberosities in our study. Cortical thickness varies along the length of the tuberosities, and local bone microarchitecture has been shown to affect suture anchor biomechanics. ${ }^{18,19}$ Anchors may also need to be inserted at different angles in the greater and lesser tuberosities to achieve fixation, with some studies showing the insertion angle can significantly affect performance. $^{20-22}$ Yet despite the differences in anatomy and anchor biomechanics at the tuberosities, we
Table 4. Postoperative MRI Anchor Fluid Signal Comparison: Anchor Location

\begin{tabular}{|c|c|c|c|c|c|}
\hline & \multicolumn{2}{|c|}{$\mathrm{GT}(n=49)$} & \multicolumn{2}{|c|}{ LT $(n=19)$} & \multirow[b]{2}{*}{$P$} \\
\hline & $N$ & $\%$ & $n$ & $\%$ & \\
\hline Grade 0 & 25 & $51.0 \%$ & 9 & $47.4 \%$ & .787 \\
\hline Grade 1 & 19 & $38.8 \%$ & 9 & $47.4 \%$ & .518 \\
\hline Grade 2 & 5 & $10.2 \%$ & 1 & $5.3 \%$ & .519 \\
\hline \multirow[t]{2}{*}{ Grade 3} & 0 & $.0 \%$ & 0 & $.0 \%$ & na \\
\hline & Mean & S.D. & Mean & S.D. & \\
\hline Mean Grade & .59 & .67 & .58 & .59 & .954 \\
\hline
\end{tabular}

GT, greater tuberosity; LT, lesser tuberosity; $n$, number; na, not applicable; S.D., standard deviation.

Dividing line between Grades 1 and 2 represents the transition from minimal perianchor fluid signal (Grade 1) to cyst formation (Grades 2 and 3).

did not observe any difference in perianchor fluid signal between these regions.

In the current study, $89 \%$ of the all-suture anchors had no significant perianchor fluid formation at an average 20.4 months postoperatively, which is in line with prior studiers evaluating soft anchors in rotator cuff repair. Van der Bracht et al. ${ }^{12}$ evaluated the MRI perianchor fluid appearance of 47 all-suture anchors (JuggerKnot, Biomet, Warsaw, IN) used for rotator cuff repair in 20 patients at an average 1.53 years postoperatively and found that $90 \%$ of the soft anchors had no fluid signal. Ro et al. ${ }^{14}$ also found $91 \%$ of the allsuture anchors (Y-Knot RC) used for rotator cuff repair had no significant perianchor fluid signal. Our study is unique in that it compares hard and soft anchors in the same shoulder, with each patient acting as their own control. This limits the number of confounding factors that could affect perianchor fluid signal, including differences in bone quality, immune response, and healing potential.

\section{Limitations}

This study has several limitations. First, our average follow-up time of 20.4 months was relatively short. There is no agreed upon timing to obtain postoperative MRI to assess cyst formation, and our minimum l-year follow-up is consistent with similar studies using MRI to evaluate perianchor fluid signal in ARCR, ${ }^{13}$ but perianchor cysts can change over time, and our study may only represent a snapshot of an evolving process. ${ }^{23}$ Second, our study sample was relatively small, and although we imaged enough anchors to power our primary outcome measure comparing perianchor fluid signal between hard and soft anchors, our study may have been underpowered to find a difference in perianchor fluid signal between types of hard anchor (PEEK vs biocomposite) or between anchor location (greater vs lesser tuberosity). Third, although we grouped 
biocomposite and PEEK anchors into a single "hard" anchor group for comparison purposes, these anchors are made of different materials and may result in different amounts of tissue reaction and fluid signal. Fourth, out of the 15 anchors used for biceps tenodesis in our study, 3 were used for biceps tenodesis alone, while 12 were used for simultaneous biceps tenodesis and cuff repair (subscapularis or anterior supraspinatus). While the 12 anchors used for simultaneous cuff repair and biceps tenodesis could have experienced a different biomechanical environment than the 3 anchors used for tenodesis alone, which could potentially affect perianchor fluid signal, prior cadaveric analysis has found the load to failure of anchors to be similar regardless of anchor position (tuberosities vs humeral neck) or local bone microarchitecture variations. ${ }^{24}$ Fifth, our study design using soft and hard anchors in the same shoulder limits our ability to assess how fluid signal differences in one anchor type affects clinical outcomes, as it is impossible to isolate one anchor type from another anchor type to ensure no confounding interaction.

\section{Conclusions}

Hard suture anchors showed increased fluid signal compared to soft suture anchors at short-term followup after ARCR, but there was no difference in cyst formation between anchor types.

\section{References}

1. Denard PJ, Burkhart SS. The evolution of suture anchors in arthroscopic rotator cuff repair. Arthroscopy 2013;29: 1589-1595.

2. Burkhart SS, Diaz Pagàn JL, Wirth MA, Athanasiou KA. Cyclic loading of anchor-based rotator cuff repairs: Confirmation of the tension overload phenomenon and comparison of suture anchor fixation with transosseous fixation. Arthroscopy 1997;13:720-724.

3. Haneveld H, Hug K, Diederichs G, Scheibel M, Gerhardt C. Arthroscopic double-row repair of the rotator cuff: A comparison of bio-absorbable and non-resorbable anchors regarding osseous reaction. Knee Surg Sports Traumatol Arthrosc 2013;21:1647-1654.

4. Pilge H, Spang J, Rose T, Wolter H, Woertler K, Imhoff $\mathrm{AB}$. Osteolysis after rotator cuff repair with bioabsorbable anchors. Arch Orthop Trauma Surg 2012;132: 305-310.

5. Nagra NS, Zargar N, Smith RD, Carr AJ. Mechanical properties of all-suture anchors for rotator cuff repair. Bone Joint Res 2017;6:82-89.

6. Pfeiffer FM, Smith MJ, Cook JL, Kuroki K. The histologic and biomechanical response of two commercially available small glenoid anchors for use in labral repairs. J Shoulder Elbow Surg 2014;23:1156-1161.
7. Bernardoni ED, Frank RM, Veera SS, et al. Biomechanical analysis of medial-row all-suture suture anchor fixation for rotator cuff repair in a pair-matched cadaveric model. Arthroscopy 2019;35:1370-1376.

8. Ergün S, Akgün U, Barber FA, Karahan M. The clinical and biomechanical performance of all-suture anchors: A systematic review. Arthrosc Sports Med Rehabil 2020;2: e263-e275.

9. Erickson J, Chiarappa F, Haskel J, et al. Biomechanical comparison of a first- and a second-generation all-soft suture glenoid anchor. Orthop J Sports Med 2017;5: 2325967117717010.

10. Tompane T, Carney J, Wu WW, et al. Glenoid bone reaction to all-soft suture anchors used for shoulder labral repairs. J Bone Joint Surg Am 2018;100:1223-1229.

11. Willemot L, Elfadalli R, Jaspars KC, et al. Radiological and clinical outcome of arthroscopic labral repair with allsuture anchors. Acta Orthop Belg 2016;82:174-178.

12. Van der Bracht H, Van den Langenbergh T, Pouillon M, Verhasselt S, Verniers P, Stoffelen D. Rotator cuff repair with all-suture anchors: A midterm magnetic resonance imaging evaluation of repair integrity and cyst formation. J Shoulder Elbow Surg 2018;27:2006-2012.

13. Kim SH, Kim DY, Kwon JE, Park JS, Oh JH. Perianchor cyst formation around biocomposite biodegradable suture anchors after rotator cuff repair. Am J Sports Med 2015;43: 2907-2912.

14. Ro K, Pancholi S, Son HS, Rhee YG. Perianchor cyst formation after arthroscopic rotator cuff repair using allsuture-type, bioabsorbable-type, and PEEK-type anchors. Arthroscopy 2019;35:2284-2292.

15. Sugaya H, Maeda K, Matsuki K, Moriishi J. Repair integrity and functional outcome after arthroscopic double-row rotator cuff repair. A prospective outcome study. J Bone Joint Surg Am 2007;89:953-960.

16. Hedelin H, Hebelka H, Brisby H, Laine T. MRI evaluation of resorbable poly lactic-co-glycolic acid (PLGA) screws used in pelvic osteotomies in children-a retrospective case series. J Orthop Surg Res 2020;15:329.

17. Jin S, Chun YM. Peri-anchor cyst formation after arthroscopic Bankart repair: Comparison between biocomposite suture anchor and all-suture anchor. Clin Shoulder Elbow 2020;23:178-182.

18. Kholinne E, Lee HJ, Kim SJ, Park SH, Jeon IH. The relationship between age, rotator cuff integrity, and osseous microarchitecture of greater tuberosity: Where should we put anchor? Acta Orthop Traumatol Turc 2018;52:22-26.

19. Yakacki CM, Poukalova M, Guldberg RE, et al. The effect of the trabecular microstructure on the pullout strength of suture anchors. J Biomech 2010;43:1953-1959.

20. Galland A, Airaudi S, Gravier R, Le Cann S, Chabrand P, Argenson JN. Pullout strength of all suture anchors in the repair of rotator cuff tears: a biomechanical study. Int Orthop 2013;37:2017-2023.

21. Ntalos D, Sellenschloh K, Huber G, et al. Conventional rotator cuff versus all-suture anchors-A biomechanical study focusing on the insertion angle in an unlimited cyclic model. PLoS One 2019;14:e0225648. 
22. Strauss E, Frank D, Kubiak E, Kummer F, Rokito A. The effect of the angle of suture anchor insertion on fixation failure at the tendon-suture interface after rotator cuff repair: Deadman's angle revisited. Arthroscopy 2009;25:597-602.

23. Chung SW, Lee YS, Kim JY, et al. Changes in perianchor cyst formation over time after rotator cuff repair:
Influential factors and outcomes. Am J Sports Med 2019;47: 165-172.

24. Barber FA, Feder SM, Burkhart SS, Ahrens J. The relationship of suture anchor failure and bone density to proximal humerus location: A cadaveric study. Arthroscopy 1997;13:340-345. 